\title{
Mutaciones del gen RAD51 en el cáncer familiar de ovario: revisión de la literatura
}

\author{
Alexandra Madariaga L. ${ }^{1}$, Daniel E. Sanabria S. ${ }^{2}$, Luz Dary Gutiérrez C. ${ }^{a}$ \\ ${ }^{1}$ Residente, Programa de Ginecología Oncológica, Hospital de San José. ${ }^{2}$ Ginecólogo Oncólogo, Hospital de San José. \\ Bogotá, Colombia.
}

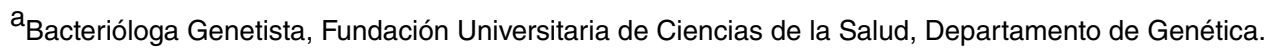

\section{RESUMEN}

Antecedentes: En los últimos 15 años, el carcinoma familiar de ovario, ha sido atribuido en su mayoría a mutaciones en BRCA 1 y 2 . Sin embargo, aproximadamente el $25 \%$ de los nuevos casos se asocian a mutaciones aisladas de genes implicados en el mecanismo de reparación del ADN por recombinación homóloga. Mutaciones monoalélicas de RAD51 han sido identificadas en pacientes con historia de carcinoma mama y ovario, tamizaje negativo para BRCA 1 y 2, y por lo menos con un caso de carcinoma de ovario en el linaje. Objetivo: Describir las mutaciones en el complejo RAD51 con el fin de identificar su papel en el cáncer de ovario familiar. Método: Se realizó una búsqueda de la literatura en bases de datos de los últimos 10 años con los siguientes términos MeSH: "RAD51", "ovarian cancer", "ovarian neoplasm", "family ovarian cancer". Resultados: Se encontró una prevalencia de la mutación en genes del complejo RAD51 que varía entre $0,2 \%$ y $2,5 \%$, según la etnia estudiada, siendo una de las causas de tumores serosos de ovario de alto grado en mujeres entre los 57 y 60 años. Conclusión: Mutaciones de RAD51 en pacientes negativas para mutaciones de BRCA 1 y 2, se asocian al síndrome familiar mama-ovario, con un aumento del riesgo para carcinoma de ovario, pero sin modificaciones para el carcinoma de mama.

\section{PALABRAS CLAVE: RAD51, cáncer de ovario, neoplasia ovárica, cáncer familiar de ovario}

\section{SUMMARY}

Background: In the last fifteen years, familiar ovarian carcinoma has been related to BRCA 1 and 2 mutations. However, $25 \%$ of new cases of ovarian neoplasm are explained by isolated genes involved in the mechanism of homologous recombination. Patients with family history of ovarian and breast carcinoma, negative for BRCA mutations and at least with one case of invasive ovarian carcinoma have been identify with monoallelic mutations in RAD51. Objective: To describe mutations on RAD51 complex, in order to identify its role in familiar ovarian cancer. Methodology: A systematic review of the literature of the last ten years involving the main data bases and using the following MeSH terms: "RAD51", "ovarian cancer", "ovarian neoplasm", "family ovarian cancer". Results: Prevalence reported for RAD51 mutation is between 0.2 and $2.5 \%$, associated with the ethnicity of the population involved. Also is considered a cause for high grade serous ovarian carcinoma in women between 57 and 60 years old. RAD51C and RAD51D germ line mutations are related to ovarian-breast hereditary syndrome, in negative population for BRCA 1 and 2 mutations. Conclusion: Patients with RAD51 mutations, negative for BRCA mutation are associated with ovarian-breast cancer syndrome increasing the risk just for ovarian cancer.

KEY WORDS: RAD51, ovarian cancer, ovarian neoplasm, familiar ovarian cancer 


\section{INTRODUCCIÓN}

El cáncer de ovario se considera responsable de aproximadamente el $6 \%$ de todos los cánceres femeninos. Corresponde al $27 \%$ de las neoplasias ginecológicas, y al $53 \%$ de las muertes femeninas por cáncer (1). En el 2012, la Sociedad Americana de Cáncer reportó 22.280 nuevos casos, y 15.500 muertes por carcinoma de ovario, convirtiéndolo en el cáncer ginecológico más letal (1-3). Mientras que la población general tiene un riesgo durante toda la vida del $1,6 \%$ de desarrollar cáncer de ovario $(4,5)$, tener uno o dos familiares en primer grado con este diagnóstico, aumenta el riesgo a un 5 y $7 \%$ respectivamente (6-8).

Se creía que por lo menos el $10 \%$ de los carcinomas de ovario se asociaban a síndromes heredados, atribuidos casi en su mayoría a mutaciones de BRCA 1 y 2. El riesgo estimado para desarrollar cáncer de ovario de alto grado a los 70 años es de $37 \%$ para mutaciones de BRCA 1 y de $21 \%$ para BRCA $2(9,10)$. Sin embargo datos recientes reportan que por lo menos el $25 \%$ de los nuevos casos de carcinoma de ovario, se asocian a mutaciones de genes aislados reparadores del ADN: BARD1, BRIP1, CHEK2, MRE11, MSH6, NBN, PALB2, RAD 50, RAD51C(11) у TP53 $(7,12)$.

La recombinación homóloga se considera un proceso celular fundamental, encargado de reparar las rupturas de la doble cadena del ADN (13). La disfunción de este mecanismo, desencadena inestabilidad genómica, llevando a la translocación cromosómica, deleción, amplificación o pérdida de la heterozigocidad, convirtiéndose en un factor de susceptibilidad para el desarrollo del cáncer $(14,15)$.

El complejo RAD51 está formado por cinco proteínas (RAD51B, RAD51C, RAD51D, XRCC2 y $X R C C 3)$, las cuales se ensamblan en polímeros que envuelven las hebras de ADN, en los puntos de ruptura, convirtiéndose en un transductor de la señalización del daño celular, catalizando el apareamiento con la hebra homóloga de ADN $(13,15)$. Adicionalmente, se describe como un activador de los punto de chequeo celular, fosforilando CHEK2 y frenando el ciclo celular en respuesta a daños del ADN (15-17).

Los niveles de expresión del gen juegan un papel central en la regulación de la recombinación homóloga. Mutaciones que reducen su actividad, aumentan la sensibilidad al daño de la doble cadena de ADN, reduciendo las tasas de reparación y aumentando la inestabilidad genómica $(15,18)$. Igualmente, se han descrito alteraciones en la concentración de RAD51, desencadenantes de resistencia a la quimio-radioterapia en los tumores de ovario de alto grado (18). Las mutaciones monoalélicas de la línea germinal de RAD51, se han visto implicadas en el desarrollo de cáncer familiar de ovario, en pacientes con tamizaje previo negativo para BRCA 1 y $2(11,18)$.
RAD51 ha sido postulado como blanco molecular orientado a la detección temprana del carcinoma de ovario, por lo tanto es necesario revisar la evidencia en la literatura universal respecto a su asociación con la aparición de cáncer familiar de ovario, con el fin de soportar la necesidad de realizar estudios de intervención en nuestra población.

\section{METODOLOGÍA}

Se realizó una revisión de la literatura registrada en las principales bases de datos; PUBMED, EMBASE, OVID, Lilacs, con los siguientes términos MeSH: "RAD51", "ovarian cancer", "ovarian neoplasm", "family ovarian cancer", la cual se limitó a los artículos que describen mutaciones en humanos, de los últimos 10 años, en inglés y español. Adicionalmente se realizó una búsqueda manual de los artículos relevantes no encontrados durante la búsqueda inicial, pero referidos por su importancia en los artículos principales.

De los 112 artículos arrojados, 6 fueron seleccionados al mostrar la relación entre mutaciones aisladas de RAD51 y el desarrollo de cáncer de ovario, en mujeres con antecedente familiar de carcinomas ovario y/o mama pero sin mutación de BRCA 1 y 2.

En total 17 artículos fueron incluidos y analizados posteriormente en su calidad por medio de la herramienta SIGN para estudios de cohorte y casos y controles. Como resultado de dicha evaluación, dos fueron clasificados como de alta calidad y quince de calidad aceptable. Los resultados de la estrategia de búsqueda pueden verse en la Figura 1.

\section{RESULTADOS}

Las características de los estudios incluidos dentro de la presente revisión pueden verse en la Tabla I. A continuación se presentan los resultados acorde con tópicos como, frecuencia de la mutación, característicos de la población estudiada, tipos de mutaciones encontradas, y cánceres relacionados con la mutación.

Frecuencia de mutaciones en línea germinal de RAD51 en el cáncer familiar de ovario. La tasa de mutación reportada para RAD51C en familias con historia de carcinoma mama y ovario varía entre $0,2 \%(19)$ y $2,5 \%$ (20), mientras que para RAD51D se encuentran entre $0,6 \%(21)$ y $2,9 \%(22)$.

Entre los estudios de alta calidad, Pelttari y cols posiciona a RAD51C como gen de susceptibilidad para cáncer de ovario; concluyen que la asociación de la mutación en familias con historia de cáncer mama/ovario, se debe a un aumento del riesgo para cáncer de ovario, mas no para el cáncer de mama. Evaluaron 277 pacientes con cáncer familiar de mama/ovario, donde reportan la mutación en el $2 \%$ de las familias con cáncer mama/ovario (23). 


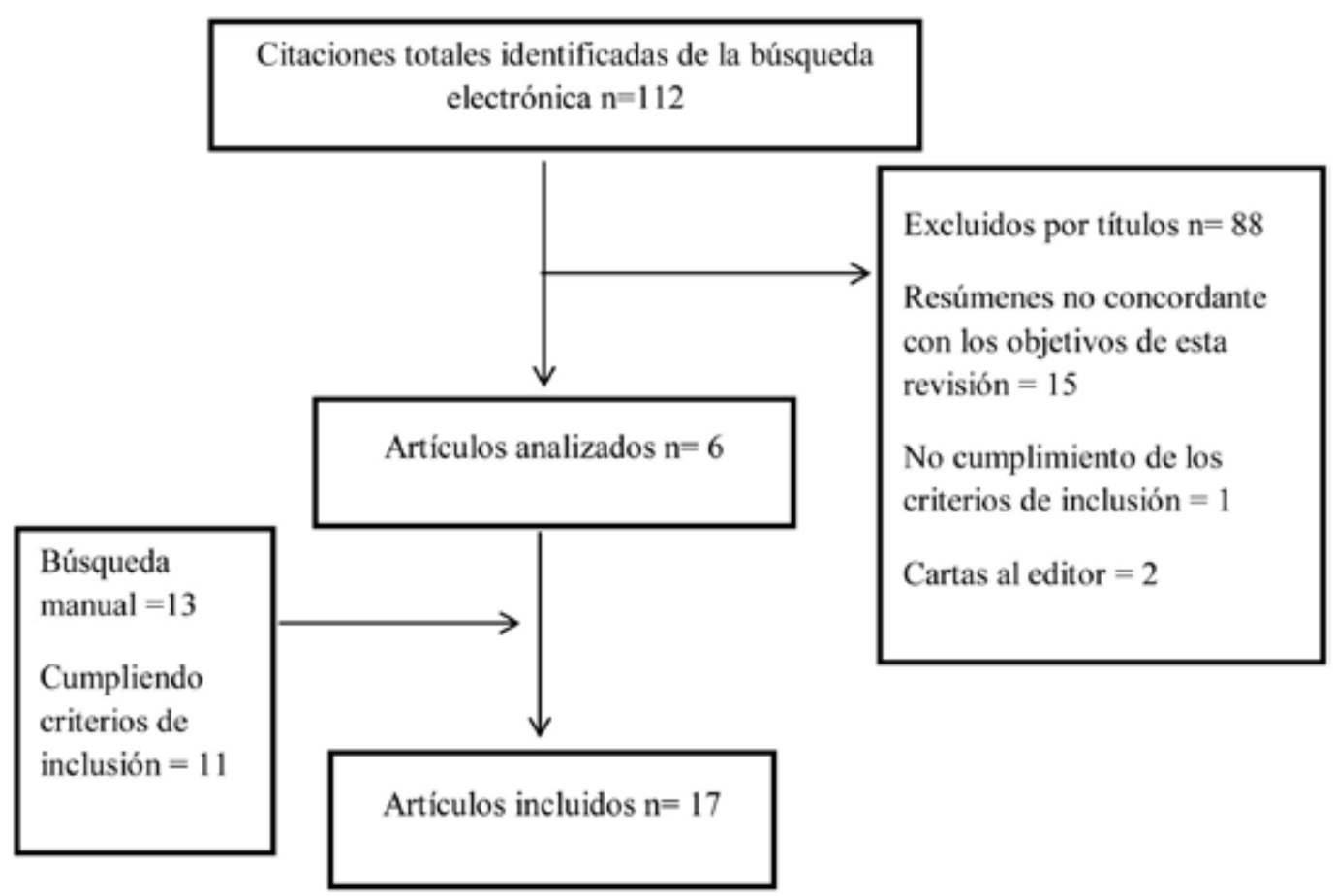

Figura 1. Diagrama de resultados identificados para la revisión.

Tabla I

CARACTERÍSTICAS DE LOS ARTÍCULOS INCLUIDOS EN LA REVISIÓN

\begin{tabular}{|c|c|c|c|c|}
\hline Gen & $\begin{array}{c}\text { Carcinoma de } \\
\text { ovario }\end{array}$ & Carcinoma de mama & $\begin{array}{l}\text { Carcinoma de } \\
\text { ovario y mama }\end{array}$ & Tipo de tumores \\
\hline [22]RAD51D & $5 / 707$ & $2 / 2015$ & $3 / 105$ & $\begin{array}{l}\text { CM: Ductal, CO: } \\
\text { Seroso }\end{array}$ \\
\hline [21]RAD51D & $\begin{array}{l}\text { RR } 6.3 ; \text { IC } 95 \% \\
2.86-13.8\end{array}$ & RR 1.32; IC95\% 0.59-2.96 & Sin información & $\begin{array}{l}\text { CO: serosos/en- } \\
\text { dometroide. CM: } \\
\text { ductal/medular }\end{array}$ \\
\hline [34]RAD51D & $1 \%$ & $0 \%$ & $0.83 \%$ & $\begin{array}{l}\text { CO: seroso/ endo- } \\
\text { metroide }\end{array}$ \\
\hline [33]RAD51C & Sin información & Sin información & $1 / 147(1 / 35 ; 2.9 \%)$ & Sin información \\
\hline [11]RAD51C & Sin información & Sin información & $1.3 \%$ & $\begin{array}{l}\mathrm{CM} \text { : ductal, CO: } \\
\text { serosos/endome- } \\
\text { troide }\end{array}$ \\
\hline [28]RAD51 & Sin información & Sin información & Sin información & Sin información \\
\hline [32]RAD51C & $0.6 \%$ & Sin información & Sin información & CO: seroso-papilar \\
\hline [19]RAD51C & Sin información & Sin información & Sin información & Sin información \\
\hline [20]RAD51C & Sin información & Sin información & $\begin{array}{l}\text { Tasa de mutación } \\
2.5 \%\end{array}$ & $\begin{array}{l}\text { CO: seroso/endo- } \\
\text { metroide }\end{array}$ \\
\hline [24]RAD51C & 157 con HF de CM & $\begin{array}{l}35 \text { con HF de Ca ovario/ } \\
\text { mama }\end{array}$ & Sin información & Sin información \\
\hline
\end{tabular}




\section{CONTINUACION TABLA I}

\begin{tabular}{|c|c|c|c|c|}
\hline Gen & $\begin{array}{l}\text { Carcinoma de } \\
\text { ovario }\end{array}$ & Carcinoma de mama & $\begin{array}{l}\text { Carcinoma de } \\
\text { ovario y mama }\end{array}$ & Tipo de tumores \\
\hline [31]RAD51C & $1 / 438$ con HF de CM & $1.3 \% ; 4 / 300$ & Sin información & $\begin{array}{l}\text { CO: Serosos de alto } \\
\text { grado, CM: ductal }\end{array}$ \\
\hline [30]RAD51C & Sin información & $2 / 34$ & Sin información & $\begin{array}{l}\text { CM: Ductal, CO: } \\
\text { seroso papilar }\end{array}$ \\
\hline [27]RAD51C & Sin información & Sin información & Sin información & Sin relación \\
\hline [23]RAD51C & $\begin{array}{l}\text { CM familiar } 0.2 \% \\
\text { VS. } 0.1 \% \text { CM } \\
\text { aislado }\end{array}$ & $\begin{array}{l}1 \% . \text { Prevalencia global } \\
\text { para CO } 1.4 \%, \text { OR } 9.37 \\
(1,87-46.4 ; \mathrm{P}=0.004), \\
\text { Mutaciones ante HF } \\
25 \% \text {, OR } 33.8(5.15-221 ; \\
\mathrm{P}=0.05)\end{array}$ & $\begin{array}{l}\text { Familias de } \\
\text { alto riesgo:OR } \\
13.59 \text { (1.89-97.6; } \\
\mathrm{P}=0.026), \mathrm{CO} \text { fa- } \\
\text { miliar aislado OR } \\
213 \text { (25.6-1769; } \\
\mathrm{P}=0.033)\end{array}$ & $\begin{array}{l}\mathrm{CM} \text { : ductal/lobular. } \\
\mathrm{CO} \text { : serosos/endo- } \\
\text { metroide }\end{array}$ \\
\hline [29]RAD51D & Sin información & $\begin{array}{l}\text { 1/51 (HF } \geq 2 \text { casos), } \\
1 / 124 \text { ( } \mathrm{HF} \text { de } 1 \text { caso) }\end{array}$ & Sin información & $\begin{array}{l}\text { CM: ductal, CO: cé- } \\
\text { lulas claras, seroso } \\
\text { de alto grado }\end{array}$ \\
\hline [26]RAD51C & Sin información & Sin información & Sin información & Sin información \\
\hline [25]RAD51C & Sin información & Sin información & Sin información & Sin información \\
\hline
\end{tabular}

CO: Carcinoma de ovario. CM: Carcinoma mamario. HF: Historia familiar.

Por otro lado, Lu y cols en el 2012, reportan un estudio de cohorte, donde se analizan los resultados del análisis mutacional de RAD51C en 192 familias con alto riesgo de carcinoma mama y/u ovario; este estudio no logró identificar ninguna mutación, atribuyendo este resultado al número limitado de familias con cáncer de mama y ovario $(n=35)(24)$.

Para los artículos de calidad aceptable, cuatro no mostraron una relación directa entre la mutación del gen y la enfermedad (25-28), los restantes encontraron mutaciones en las familias con carcinoma de mama y ovario, pero no en los casos exclusivos de carcinoma de mama $(11,19-22,29-34)$, relacionando la presencia de mutaciones en los casos con historia familiar de por lo menos un caso de carcinoma de ovario: OR: 13,59; IC95\% 1,89-97,6; $\mathrm{p}=0,026$ en los casos de cáncer familiar de mama y ovario, y OR: 213; IC95\% 25,6-1769; $p=0,0002$ en casos de cáncer familiar de ovario en ausencia de cáncer de mama (23).

Todos los estudios para RAD51D, de calidad aceptable, postulan a este gen como factor asociado al carcinoma de ovario. Loveday y cols, lograron mostrar que las mutaciones de RAD51D, pueden llegar aumentar hasta 6 veces el riesgo de carcinoma de ovario (RR 6,3; IC95\% 2,86-13,85), sin afectar, o con un aumento muy sutil en el riesgo de cáncer de mama (RR 1,32; IC95\% 0,59-2,96) (21). Caracterización de la población estudiada. Los 17 artículos incluidos y revisados, incluyeron poblaciones de diversas etnias; Finlandia $(22,23,28,33)$, Estados Unidos $(24,27,30,34)$, España $(19,31)$, Reino Unido (21), Alemania (11), Australia (32), Francia (20), Israel (26), Canadá, Bélgica y Países bajos (25). Esta diversidad poblacional, se plantea como la causa principal del amplio rango de prevalencias reportada.

De la población con diagnóstico de cáncer de ovario con mutaciones en RAD51, se reporta una edad media de diagnóstico para cáncer de mama y ovario de 48,9 años (rango: 40-60,1 años) y 56 años (rango: 46-67,5 años), respectivamente. Respecto a los tipos histológicos más comunes se reporta en mama el carcinoma ductal infiltrante, y en ovario el carcinoma seroso papilar de alto grado, el endometroide, y el de células claras. Wickramanyake y cols reportan inclusive un caso de carcinomas sincrónicos endometroides de ovario y endometrio (34).

Mutaciones más frecuentes en RAD51 que confieren susceptibilidad para el desarrollo de cáncer de ovario familiar (Tabla II). Las mutaciones más comunes de la línea germinal de 
RAD51 asociadas a carcinoma familiar de ovario son de tipo, corte y empalme, corrimiento del marco de lectura y sin sentido, las cuales generan proteínas truncadas. Se postula RAD51 como gen de susceptibilidad para cáncer de ovario de penetrancia moderada-alta ante antecedente familiar de la enfermedad $(21,22)$.
Meindly cols en el 2010, publica las primeras 6 mutaciones más comunes, marcando la posible relación de la mutación de RAD51C con el síndrome familiar mama/ovario. Las 6 mutaciones patógenas reportadas en este estudio son: 224_225insA, 525_526insC, 145+1G>T, 904+5G>T, G125V, L138F (11).

Tabla II

MUTACIONESY POLIMORFISMOS REPORTADOS EN LOS ARTÍCULOS INCLUIDOS

\begin{tabular}{|c|c|c|c|}
\hline Gen & Tipo de mutación & Mutación & Polimorfismo \\
\hline $\begin{array}{l}\text { RAD51D } \\
\text { [22] }\end{array}$ & Corte y empalme & c. $576+1 \mathrm{G}>\mathrm{A}$ & $\begin{array}{l}\text { rs28363258, C. } 82+128 \mathrm{C}>\mathrm{T} \text {, } \\
\text { rs9901455, p.Ser78Ser, } \\
\text { rs4796033. p.Arg165Gln, } \\
\text { rs45496096, C.567+53C>T }\end{array}$ \\
\hline $\begin{array}{l}\text { RAD51D } \\
\text { [21] }\end{array}$ & $\begin{array}{l}\text { Cambios en el marco de } \\
\text { lectura por deleción o in- } \\
\text { serción, sin sentido, corte } \\
\text { y empalme. }\end{array}$ & $\begin{array}{l}\text { c.363delA, , c.803G>A; } \\
\text { p.Trp268X, c.556C>T; } \\
\text { p.Arg186X, c. } 480+1 G>A \text {, } \\
\text { c.345G>C; p.GIn115Hisc, } \\
\text { c.757C>T; p.Arg253X } \\
\text { c.270_271dupTA }\end{array}$ & $\begin{array}{l}\text { rs9901455, p.Ser78Ser, } \\
\text { rs4796033, p.Arg165Gln, } \\
\text { rs28363284, p.Glu233Gly }\end{array}$ \\
\hline $\begin{array}{l}\text { RAD51D } \\
{[34]}\end{array}$ & $\begin{array}{l}\text { Sin sentido, cambios en el } \\
\text { marco de lectura, corte y } \\
\text { empalme }\end{array}$ & $\begin{array}{l}\text { c.580delA, p.T194L_fsX6, } \\
\text { ( Chr17:33,430,560) } \\
\text { c.694C>T, p.R232X, } \\
\text { (chr17:33,430,296) } \\
\text { c.131_144+24del38^, } \\
\text { p.V28G_fsX21, } \\
\text { (chr17:33,446,115) }\end{array}$ & $\begin{array}{l}\text { c.26G>C, p.C9S, } \\
\text { (chr17:33,446,607), } \\
\text { c.137C>G, p.S46C, } \\
\text { (chr17:33,446,137), c.620C>T, } \\
\text { p.S207L, (chr17:33,430,520), } \\
\text { c.715C>T, p.R239W, } \\
\text { (chr17:33,430,317), c.932T>A, } \\
\text { p.1311N, (chr17:33,428,027) }\end{array}$ \\
\hline $\begin{array}{l}\text { RAD51C } \\
{[33]}\end{array}$ & $\begin{array}{l}\text { Cambios en el marco de } \\
\text { lectura }\end{array}$ & c.-13_14del27, c.774delT & No polimorfismo \\
\hline $\begin{array}{l}\text { RAD51C } \\
{[11]}\end{array}$ & $\begin{array}{l}\text { Inserción, cambios en el } \\
\text { marco de lectura, sentido } \\
\text { erróneo }\end{array}$ & $\begin{array}{l}\text { 224_225insA, } \\
\text { 525_526insC, 145+1G>T, } \\
904+5 G>T, G 125 V, L 138 F\end{array}$ & No polimorfismo \\
\hline $\begin{array}{l}\text { RAD51C } \\
{[32]}\end{array}$ & $\begin{array}{l}\text { Sin sentido } \\
\text { Cambios en el marco de } \\
\text { lectura. }\end{array}$ & $\begin{array}{l}\text { c.397C>T, p.Gln133X, } \\
\text { c.68_72 dup, } \\
\text { p.Val25CysfsX3 } \\
\text { p.Gly162Glu, } \\
\text { p.GIn178Pro, p.Gly264Ser, } \\
\text { p.Thr287Ala }\end{array}$ & No polimorfismo \\
\hline $\begin{array}{l}\text { RAD51C } \\
{[19]}\end{array}$ & $\begin{array}{l}\text { Cambios en el marco de } \\
\text { lectura }\end{array}$ & c c.774delT, p.Arg258fs & $\begin{array}{l}\text { c. } 904+34 \text { T>C. c. } 859 A>G \text {, } \\
\text { p.Thr287Ala }\end{array}$ \\
\hline
\end{tabular}




\section{CONTINUACION TABLA II}

\begin{tabular}{|c|c|c|c|}
\hline Gen & Tipo de mutación & Mutación & Polimorfismo \\
\hline $\begin{array}{l}\text { RAD51C } \\
\text { [20] }\end{array}$ & $\begin{array}{l}\text { Sin sentido, corte y } \\
\text { empalme }\end{array}$ & $\begin{array}{l}\text { c. } 577 \mathrm{C}>\mathrm{T}, \text { p.Arg193X, } \\
\text { c.965+5A }>\mathrm{G}, \\
\text { p.Glu303TrpfsX41, c.905- } \\
\text { 2A>C, p.Glu303TrpfsX41 }\end{array}$ & $\begin{array}{l}\text { rs12946397, c. } 1-26 \mathrm{C}>\mathrm{T}, \\
\text { rs61758784, c. } 376 \mathrm{G}>\mathrm{A}, \\
\mathrm{rs} 28363317, \mathrm{c} .859 \mathrm{~A}>\mathrm{G}, \\
\mathrm{rs} 28363318, \mathrm{c} .904+34 \mathrm{~T}>\mathrm{C}\end{array}$ \\
\hline $\begin{array}{l}\text { RAD51C } \\
\text { [31] }\end{array}$ & $\begin{array}{l}\text { Sentido erróneo, proteína } \\
\text { truncada }\end{array}$ & $\begin{array}{l}\text { c.414G.C; p.Leu138Phe, } \\
\text { c.774delT; p.Arg258fs, } \\
\text { c.404G.A, p.Cys135Tyr, } \\
\text { c.428A.G, p.Gln143Arg, } \\
\text { c.656T.C, p.Leu219Ser }\end{array}$ & No polimorfismo \\
\hline $\begin{array}{l}\text { RAD51C } \\
\text { [30] }\end{array}$ & $\begin{array}{l}\text { Sentido erróneo (variantes } \\
\text { no patogénicas) }\end{array}$ & G513D, R214C & T287A, A126T \\
\hline $\begin{array}{l}\text { RAD51C } \\
\text { [23] }\end{array}$ & $\begin{array}{l}\text { Proteína truncada, corte y } \\
\text { empalme }\end{array}$ & c.93delG, c. $837+1 G>A$ & $\begin{array}{l}\text { c. }-26 C . T 175, \text { c. } 93 \text { delG } 275 \text {, } \\
\text { c. } 790 G>A, c .837+1 G>A \text {, } \\
\text { rs } 12946397 \\
\text { c. } 859 A>G \text {, } \\
\text { rs } 28363317 . c .904+34 T>C \text {, } \\
\text { rs } 28363318\end{array}$ \\
\hline $\begin{array}{l}\text { RAD51D } \\
\text { [29] }\end{array}$ & $\begin{array}{l}\text { Sin sentido, sustitución en } \\
\text { intrón, creación de sitio } \\
\text { aceptor, sinónima }\end{array}$ & p.Arg186X, c.556C>T & $\begin{array}{l}\text { p.Cys119Arg, c.355T>C, } \\
\text { p.Gly265Arg, c.793G>A, } \\
\text { c.83-26A>G (intron 1), } \\
\text { r.145_263del;p.Ala49Ser- } \\
\text { fsX2, p.Ser78Ser, c.234C4T, } \\
\text { rs9901455, p.Arg165Gln, } \\
\text { c.494G4A, rs4796033, } \\
\text { p.Glu233Gly, c.698A4G, } \\
\text { rs28363284 }\end{array}$ \\
\hline
\end{tabular}

Pelttari y cols reportan para el gen RAD51 la mutación c.576+1G>A en el 2,9\% de las pacientes con historia familiar de cáncer de mama/ovario (OR 37,82; IC95\% 3,90-366,91; $p=0,0016$ ), y entre los pacientes con historia personal o familiar de cáncer de ovario (OR 9,16; IC95\% 1,07-78,56; $p=0,024$ ), pero con frecuencias de la mutación entre pacientes con casos de carcinoma de mama similares a la de la población control $(0,1 \%$ vs. $0,05 \% ; p=1)(22)$.

Vuorela y cols en 2011, reportan dos mutaciones con características patogénicas en el gen RAD51C: la mutación c.-13_14del27 en un caso de cáncer de seno familiar y la mutación c.774delT en un caso de cáncer de ovario aislado, lo que confirma a este gen como un factor de susceptibilidad para el desarrollo de cáncer de ovario y mama, sin embargo debido a la baja frecuencia y los reportes de la mutación c.774delT esta variante podría tener un efecto fundador en la población estudiada (33).

Otros cánceres encontrados en familias con mutaciones en RAD51. Nueve de los diecisiete estudios incluidos, reportan carcinomas diferentes al de mama y/u ovario presentes en familias portadoras de mutaciones en genes del complejo RAD51 (11,19-21,23,29,31-33), uno descarta la asociación entre mutaciones a otro tipo de cáncer (22), los restantes, no aportan información (24-28,30,34). Entre las neoplasias encontradas se incluyen: carcinoma colo-rectal, pulmón, próstata, linfoma no Hodgkin, riñón, páncreas, primario desconocido, laringe, sistema nervioso central, endometrio, leucemia, hígado, melanoma, y lengua. Es importante resaltar que el carácter patogénico de la mutación ante estos cánceres es desconocido. 


\section{DISCUSIÓN}

En los últimos 15 años, el carcinoma familiar de ovario, ha sido atribuido en su mayoría a mutaciones en los genes de susceptibilidad para el carcinoma mama/ovario, BRCA 1 y 2 (5), documentándose mutaciones en un $13-15 \%$ en casos de carcinomas invasores (5,7). Recientemente RAD51C y RAD51D, quienes comparten con BRCA 1 y 2 , un papel fundamental en el mecanismo de reparación del ADN por recombinación homóloga, han sido postulados como genes asociados al carcinoma familiar de ovario (5).

Acorde a los estudios que muestran la asociación entre la mutación y el carcinoma familiar de ovario, se encuentran 8 estudios de casos y controles y 4 estudios de cohortes, solo uno de estos de alta calidad.

Se concluye que las mutaciones de RAD51C han sido reportadas en el $1,3 \%$ de familias alemanas negativas para mutaciones de BRCA, con síndrome familiar de cáncer mama/ovario, sin reportarse relación de la mutación en familias con casos exclusivos de cáncer de mama $(11,23,35)$.

Se considera de importancia, al igual que para las mutaciones de BRCA 1 y 2, la influencia del grupo étnico en la prevalencia y distribución de las mutaciones de RAD51, reportándose por lo tanto, intervalos amplios de prevalencia entre las poblaciones incluidas y mutaciones con posible efecto fundador en el gen RAD $51 \mathrm{C}$ en poblaciones de descendencia sueca descritas por Vuorela, Romero y Osorio: c.774delT (p.Arg258fs), p.Leu138Phe; y de RAD51D citadas por Osher y Loveday: p.Arg186X (c.556C>T), en población británica.

RAD51D y RAD51C se pueden postular, como genes de susceptibilidad para carcinoma de ovario de penetrancia moderada - alta, con un patrón de segregación completo, asociado a carcinomas epiteliales de ovario de alto grado, y de tipos histológicos adversos, incluyendo carcinomas de células claras, serosos y endometroides de alto grado, haciéndose manifiesto en mujeres con una edad media de 56 años.

Diferente a lo que ocurre con las mutaciones de BRCA, donde se aumenta el riesgo tanto para los carcinomas de ovario como los de mama, las mutaciones de RAD51 aumentan el riesgo de carcinoma de ovario, influenciando la aparición de carcinoma de mama solo en casos de historia familiar de carcinoma de ovario.

Respecto a los estudios donde no se logra evidenciar una asociación directa entre mutaciones en genes RAD51 y carcinoma de ovario, encontramos dos estudios de casos y controles y tres estudios de cohorte, siendo solo uno de estos últimos de alta calidad. Estos cinco estudios logran identificar mutaciones del gen, pero ninguna causante de la generación de proteínas truncadas, ni de inactivación proteica del gen.

Al haberse sugerido que la mutación de RAD51 se asocia a un mayor riesgo de carcinoma de ovario, cuatro de los cinco estudios resaltan su limitante respecto al número limitado de familias con antecedente de carcinoma de mama y ovario, como explicación plausible de la ausencia de mutación inactiva de RAD51C. Adicional a esto, se resalta lo raro de la mutación (27) como causas directas de la no asociación del gen como marcador de susceptibilidad para cáncer de mama y ovario, igualmente se resalta la necesidad de un estudio multiétnico, con grandes poblaciones para lograr definir el espectro de la mutación del gen (26).

La identificación de mutaciones que predispongan a un mayor riesgo de carcinoma de ovario, en este caso, mutación de la línea germinal RAD51, permite que mujeres portadoras consideren estrategias adecuadas reductoras del riesgo de enfermedad y muerte, por ejemplo: la salpingo-ooforectomía profiláctica $(4,5)$.

Adicionalmente, con la respuesta clínica en pacientes con carcinoma de mama, BRCA positivas, y progresión de su enfermedad luego de quimioterapias basadas en platino, con el uso de los inhibidores de la PARP (poli ADP-ribosa), se propone la identificación de biomarcadores, RAD51, para la detección de tumores con deficiencia en la recombinación homóloga, ya que estos pueden considerarse como de mayor susceptibilidad al uso de este tipo de medicamentos, permitiendo terapias dirigidas alternas a la quimioterapia $(36,37)$.

\section{CONCLUSIONES}

Mutaciones germinales de RAD51C y RAD51D, en pacientes negativas para mutaciones de BRCA 1 y 2 , se asocian al síndrome familiar mama-ovario, con un aumento del riesgo para carcinoma de ovario, pero sin modificaciones para el carcinoma de mama. Se requieren estudios multicéntricos que permitan aclarar el riesgo para cáncer de ovario, para poder plantear estrategias de tamizaje aplicables.

Agradecimientos: A la Doctora Ingrid Arévalo, Coordinador Centro Cochrane Colaborador, Fundación Universitaria de Ciencias de la Salud, Hospital San José \& Hospital Infantil de San José, por el direccionamiento en la estrategia de búsqueda, metodología y el uso de la herramienta SIGN. 


\section{REFERENCIAS}

1. Prat J. Ovarian carcinomas: five distinct diseases with different origins, genetic alterations, and clinicopathological features. Virchows Arch 2012;460(3):237-49.

2. Yoshida S, Furukawa N, Haruta S, Tanase Y, Kanayama S, Noguchi T, et al. Expression profiles of genes involved in poor prognosis of epithelial ovarian carcinoma: a review. Int J Gynecol Cancer 2009;19(6):9927.

3. Goff BA. Ovarian cancer: screening and early detection. Obstet Gynecol Clin North Am 2012;39(2):18394.

4. Folkins AK, Longacre TA. Hereditary gynaecological malignancies: advances in screening and treatment. Histopathology 2013;62(1):2-30.

5. Pennington KP, Swisher EM. Hereditary ovarian cancer: beyond the usual suspects. Gynecol Oncol 2012;124(2):347-53.

6. Hunn J, Rodriguez GC. Ovarian cancer: etiology, risk factors, and epidemiology. Clin Obstet Gynecol 2012;55(1):3-23.

7. Weissman SM, Weiss SM, Newlin AC. Genetic testing by cancer site: ovary. Cancer J 2012;18(4):320-7.

8. Kurman RJ, Shih IM. The origin and pathogenesis of epithelial ovarian cancer: a proposed unifying theory. Am J Surg Pathol 2010;34(3):433-43.

9. Kurman RJ, Shih IM. Pathogenesis of ovarian cancer: lessons from morphology and molecular biology and their clinical implications. Int J Gynecol Pathol 2008;27(2):151-60.

10. Al-Niaimi AN, Ahmed M, Petersen CB, Epithelial ovarian cancer. Obstet Gynecol Clin North Am 2012;39(2):269-83.

11. Meindl A,Hellebrand H, Wiek C, Erven V, Wappenschmidt B, Niederacher D, et al. Germline mutations in breast and ovarian cancer pedigrees establish RAD51C as a human cancer susceptibility gene. Nat Genet 2010;42(5):410-4.

12. Walsh T,Casadei S, Lee MK, Pennil CC, Nord AS, Thornton AM, et al. Mutations in 12 genes for inherited ovarian, fallopian tube, and peritoneal carcinoma identified by massively parallel sequencing. Proc Natl Acad Sci U S A 2011;108(44):18032-7.

13. Vaz F,Hanenberg H, Schuster B, Barker K, Wiek C, Erven V, et al. Mutation of the RAD51C gene in a Fanconi anemia-like disorder. Nat Genet 2010;42(5):4069.

14. Somyajit K, Subramanya S, Nagaraju G. RAD51C: a novel cancer susceptibility gene is linked to Fanconi anemia and breast cancer. Carcinogenesis 2010;31(12):2031-8.

15. Suwaki N, Klare K, Tarsounas M. RAD51 paralogs: roles in DNA damage signalling, recombinational repair and tumorigenesis. Semin Cell Dev Biol 2011;22(8):898-905.

16. Ballinger LL. Hereditary gynecologic cancers: risk assessment, counseling, testing and management. Obstet Gynecol Clin North Am 2012;39(2):165-81.

17. Shulman LP. Hereditary breast and ovarian cancer (HBOC): clinical features and counseling for BRCA1 and BRCA2, Lynch syndrome, Cowden syndrome, and Li-Fraumeni syndrome. Obstet Gynecol Clin North Am 2010;37(1):109-33, Table of Contents.
18. Klein HL. The consequences of Rad51 over expression for normal and tumor cells. DNA Repair (Amst) 2008;7(5):686-93.

19. Romero A, Pérez-Segura P, Tosar A, García-Saenz $\mathrm{JA}$, Díaz-Rubio $\mathrm{E}$, Caldés $\mathrm{T}$, et al. A HRM-based screening method detects RAD51C germ-line deleterious mutations in Spanish breast and ovarian cancer families. Breast Cancer Res Treat 2011;129(3):93946.

20. Coulet $F$, Fajac A, Colas $C$, Eyries $M$, Dion-Minière A, Rouzier R, et al. Germline RAD51C mutations in ovarian cancer susceptibility. Clin Genet 2013;83(4):3326.

21. Loveday C, Turnbull C, Ramsay E, Hughes D, Ruark $\mathrm{E}$, Frankum JR, et al. Germ line mutations in RAD51D confer susceptibility to ovarian cancer. Nat Genet 2011;43(9):879-82.

22. Pelttari LM, Kiiski J, Nurminen R, Kallioniemi A, Schleutker J, Gylfe A, et al. A Finnish founder mutation in RAD51D: analysis in breast, ovarian, prostate, and colorectal cancer. J Med Genet 2012;49(7):42932.

23. Pelttari LM, Heikkinen T, Thompson D, Kallioniemi A, Schleutker J, Holli K, et al. RAD51C is a susceptibility gene for ovarian cancer. Hum Mol Genet 2011;20(16):3278-88.

24. Lu W, Wang $X$, Lin $\mathrm{H}$, Lindor NM, Couch FJ, et al. Mutation screening of RAD51C in high-risk breast and ovarian cancer families. Fam Cancer 2012;11(3):3815.

25. De Leeneer K, Van Bockstal M, De Brouwer S, Swietek N, Schietecatte $\mathrm{P}$, Sabbaghian N, et al. Evaluation of RAD51C as cancer susceptibility gene in a large breast-ovarian cancer patient population referred for genetic testing. Breast Cancer Res Treat 2012;133(1):393-8.

26. Kushnir A, Laitman Y, Shimon SP, Berger R, Friedman $\mathrm{E}$, et al. Germline mutations in RAD51C in Jewish high cancer risk families. Breast Cancer Res Treat 2012;136(3):869-74.

27. Zheng Y, Zhang J, Hope K, Niu Q, Huo D, Olopade OI, et al. Screening RAD51C nucleotide alterations in patients with a family history of breast and ovarian cancer. Breast Cancer Res Treat 2010;124(3):85761.

28. Rapakko K, Heikkinen K, Karppinen SM, Winqvist R, et al. Screening for RAD51 and BRCA2 BRC repeat mutations in breast and ovarian cancer families. Cancer Lett 2006;236(1):142-7.

29. Osher DJ, De Leeneer K, Michils G, Hamel N, Tomiak $\mathrm{E}$, Poppe B, et al. Mutation analysis of RAD51D in non-BRCA1/2 ovarian and breast cancer families. $\mathrm{Br}$ J Cancer 2012;106(8):1460-3.

30. Clague J, Wilhoite G, Adamson A, Bailis A, Weitzel $\mathrm{JN}$, Neuhausen SL, et al. RAD51C germ line mutations in breast and ovarian cancer cases from highrisk families. PLoS One 2011;6(9):e25632.

31. Osorio $A$, Endt $D$, Fernández $F$, Eirich $K$, de la Hoya $\mathrm{M}$, Schmutzler $\mathrm{R}$, et al. Predominance of pathogenic missense variants in the RAD51C gene occurring in breast and ovarian cancer families. Hum Mol Genet 2012;21(13):2889-98.

32. Thompson ER, Boyle SE, Johnson J, Ryland GL, Sawyer S, Choong DY, et al. Analysis of RAD51C 
germline mutations in high-risk breast and ovarian cancer families and ovarian cancer patients. Hum Mutat 2012;33(1):95-9.

33. Vuorela M, Pylkäs K, Hartikainen JM, Sundfeldt $K$, Lindblom $A$, von Wachenfeldt Wäppling $A$, et al. Further evidence for the contribution of the RAD51C gene in hereditary breast and ovarian cancer susceptibility. Breast Cancer Res Treat 2011;130(3):1003-10.

34. Wickramanayake A, Bernier G, Pennil C, Casadei S, Agnew KJ, Stray SM, et al. Loss of function germline mutations in RAD51D in women with ovarian carcinoma. Gynecol Oncol 2012;127(3):552-5.
35. Akbari MR, Tonin P, Foulkes WD, Ghadirian P, Tischkowitz M, Narod SA, et al. RAD51C germline mutations in breast and ovarian cancer patients. Breast Cancer Res 2010;12(4):404.

36. Lopez J, Banerjee S, Kaye SB. New developments in the treatment of ovarian cancer--future perspectives. Ann Oncol 2013;24 Suppl 10:x69-x76.

37. Chen Y, Zhang L, Hao Q. Olaparib: a promising PARP inhibitor in ovarian cancer therapy. Arch Gynecol Obstet 2013;288(2): 367-74. 